\title{
Formalization of Formal Topology by means of the interactive theorem prover Matita
}

\author{
Andrea Asperti ${ }^{1}$, Maria Emilia Maietti ${ }^{2}$, Claudio Sacerdoti Coen ${ }^{1}$, \\ Giovanni Sambin ${ }^{2}$, and Silvio Valentini ${ }^{2}$ \\ 1 Department of Computer Science, University of Bologna \\ \{asperti, sacerdot\}@cs. unibo.it \\ 2 Department of Mathematics, University of Padova \\ \{maietti, sambin, silvio\}@math.unipd.it
}

The project entitled "Formalization of Formal Topology by means of the interactive theorem prover Matita" is an official bilateral project between the Universities of Padova and Bologna, funded by the former, active iiiiii .mine from march 2008 until august 2010. The project brought together and exploited the synergic collaboration of two communities of researchers, both centered around constructive type theory: $======$ from March 2008 until August 2010. The project aimed to bring together and exploit the synergic collaboration of two communities of researchers, both centered around constructive type theory: izidiziz r3432 on one side the Logic Group at the University of Padova, focused on developing formal, pointfree topology within a constructive and predicative framework; on the other side, the Helm group at the University of Bologna, developing the Matita Interactive Theorem Prover [2], a young proof assistant based on the Calculus of Inductive Constructions as its logical foundation. The idea of the project was to formalize and check the new approach to formal topology being developed in Padova by means of Matita, with the aim on one side to assess the truly foundational nature of the theoretical framework (i.e. its reduction to notions so elementary to be easily understood by an automatic device), and on the other to drive the development of Matita, testing the tool on a non trivial set of mathematical results, and addressing from an original theoretical perspective some key problems of constructive interactive proving (general recursion, extensionality, quotients, ...).

The project is a rare example of a significant collaboration between mathematicians and computer scientists in handling mathematical knowledge.

It is worth to emphasize that the interest in the formalization from the mathematical perspective is not in the automatic verification of the results (on which mathematicians are already largely confident with) but in the phenomenological goal to investigate the most natural way to organize a new foundational framework in a coherent set of interconnected components, their mutual relations and dependencies, our interaction with these representations, and their influence on the concrete mathematical experience (see [1]). Formalization is neither a goal nor a technique, but first and foremost a methodology.

The formalization work was mainly focused on Overlap Algebras [4,3], new algebraic structures designed to ease reasoning about subsets within intuitionistic logic. The main result checked in Matita [8] is the embedding of a suitable 
category of topological spaces into a category of generalized point-free topologies, which is an improvement on the usual adjunction between topological spaces and locales. The formalization of this result drove several major improvements of Matita, discussed in [8].

A major feature of type theory (and proof checking systems based on this framework) is that functions are live entities, in the sense that they can be effectively computed. However, in presence of dependent types and for consistency reasons, one has to restrict to a subset of total computable funtions (typically based on some well founded recursion principle), that prevents from programming in a truly natural functional style. For this reason, the encoding of general recursion and its (simulated) behaviour is a major topic for interactive proving. In [5], a new approach to this problem based on the use of inductively generated formal topologies is proposed. The work is based on previous results in [9], relating the notion of covering with that of well-founded part of a relation.

Another challenging problem in type theory/interactive proving is extensionality, and especially an extensional treatment of set theory with quotients. In [6], a two-level theory to formalize constructive mathematics is presented, developing ideas already outlined in [7]. One level is given by an intensional type theory, called Minimal Type Theory. This theory extends a previous version with collections. The other level is given by an extensional set theory that is interpreted in the first one by means of a quotient model. This two-level theory has two main features: it is minimal among the most relevant foundations for constructive mathematics; it is constructive thanks to the way the extensional level is linked to the intensional one which fulfills the "proofs as programs" paradigm and acts as a programming language. The possibility to integrate this two level approach in Matita is still under investigation.

The code of the formalization developed in the project can be found on-line at the address http://matita.cs.unibo.it/library.shtml, under the section "The Basic Picture".

\section{References}

1. Andrea Asperti and Jeremy Avigad. Zen and the art of formalization. Mathematical Structures in Computer Science, 21 (to appear), 2011.

2. Andrea Asperti, Claudio Sacerdoti Coen, Enrico Tassi, and Stefano Zacchiroli. User interaction with the Matita proof assistant. Journal of Automated Reasoning, 39(2):109-139, 2007.

3. Francesco Ciraulo, Maria Emilia Maietti, and Paola Toto. Constructive version of boolean algebra. IGPL (to appear), 2011.

4. Francesco Ciraulo and Giovanni Sambin. The overlap algebra of regular opens. Journal of Pure and Applied Algebra, 214:1988-1995, 2010.

5. Claudio Sacerdoti Coen and Silvio Valentini. General recursion and formal topology. In Proceedings Workshop on Partiality and Recursion in Interactive Theorem Provers, volume 43 of EPTCS, pages 65-75, 2010. 
6. Maria Emilia Maietti. A minimalist two-level foundation for constructive mathematics. Annals of Pure and Applied Logic, 160(3):319-354, 2009.

7. Maria Emilia Maietti and Giovanni Sambin. From Sets and Types to Topology and Analysis, Toward A Minimalist Foundation for Constructive Mathematics, chapter 6. Oxford University Press, 2005.

8. Enrico Tassi and Claudio Sacerdoti Coen. Formalizing overlap algebras in matita. Mathematical Structures in Computer Science, 21 (to appear), 2011.

9. Silvio Valentini. Cantor theorem and friends, in logical form. Annals of pure and applied logic., 163 (to appear), 2011. 